\title{
Development of a Yoruba Bible Native Application for Smartphones
}

\author{
Obe Olumide O. \\ Department of Computer \\ Science \\ Federal University of \\ Technology Akure, Nigeria
}

\author{
Fakuade Segun O. \\ Department of Computer \\ Science \\ Federal University of \\ Technology Akure, Nigeria
}

\author{
Ogundele Tunde J. \\ Department of Computer \\ Science \\ Federal University of \\ Technology Akure, Nigeria
}

\begin{abstract}
There are lots of bibles in different versions and languages and with the level of sophistication in today Smartphones; there are major increase in the development of different native apps that are widely used on these numerous smartphones. These include bible native applications in different versions and languages except Yoruba traditional language. This paper focus on the development of a complete indigenous Yoruba Language bible that will run as a native application on smartphone, the development methodology utilize the Windows phone SDK, Microsoft C\#.net and Extensible Application Markup Language (XAML). It also comprises of the architectural design of the development of native application, project architecture and application layout (The entire data, application resources and presentation layer of the application reside on the device). The application is expected to provide good and ease accessibility to the bible in an indigenous language and this will be of maximum importance to Yoruba communities, schools and churches.
\end{abstract}

\section{General Terms}

App Development, Mobile Computing, et. al.

\section{Keywords}

Language, native app; smartphone; yoruba; application; xaml.

\section{INTRODUCTION}

The evolution of smartphone all began with one famous man called Alexander Bell, it was a revolution in 1878 when he made his first phone call. Moreover, the world experienced a great concept in 1974 when a Greek man called Theodore Parakevakos patented his brian child idea behind a basic smart phone. The term smartphone did not appear until 1997, when
Erricsson describe its GS 88 "Penelope" concept as a smartphone [1] and 2008 unleashed the world to a greater sophisticated smartphones operating systems likes Windows Phones OS, iOS, Android OS and etc.

The term Native in computing means the act of written application specifically for a particular processor or mobile operating software. These apps most time are downloaded directly unto the smartphone and installed on the device. Upon initialization, the native app interfaces directly with the mobile operating system, without any intermediary or container. The native app is free to access all of the APIs that are made available by the OS vendor and, in many cases, has unique features and functions that are typical of that specific mobile OS

The windows phone architecture deployed in this word is described in figure 1. The top of the stack is made-up of two models; the Task Host, it represents the Extensible Application Markup Language (XAML) app model, which is the primary model of Windows Phone 7. Core Application is a newly introduced app model for Windows Phone, an integral part of the new Windows 8 app model [2]; this model only supports pure native apps using Direct3D for User interface in the new Windows phone 8 releases.

In this paper, a description about the development of indigenous Yoruba Language bible that will run as a native application on smartphone and also, the architectural design of development of this app. The rest of the paper is organized as follows: section II discusses the proposed scheme, section III is on contribution to knowledge and recommendation while limitation of the research and the screenshots of the application is presented in section IV and V respectively. 


\begin{tabular}{|l|l|}
\hline TaskHost \\
\hline \multicolumn{1}{|c|}{ Managed App } \\
\hline $\begin{array}{c}\text { Managed Frameworks } \\
\left.\text { (Microsoft.* \& System. }{ }^{\star}\right)\end{array}$ & $\begin{array}{c}\text { Windows Runtime } \\
\left.\text { Frameworks (Windows. }{ }^{\star}\right)\end{array}$ \\
\hline
\end{tabular}

\begin{tabular}{|l|}
\hline CoreApplication \\
\hline Native App \\
\hline $\begin{array}{c}\text { Windows Runtime } \\
\text { Frameworks (Windows.*) }\end{array}$ \\
\hline
\end{tabular}

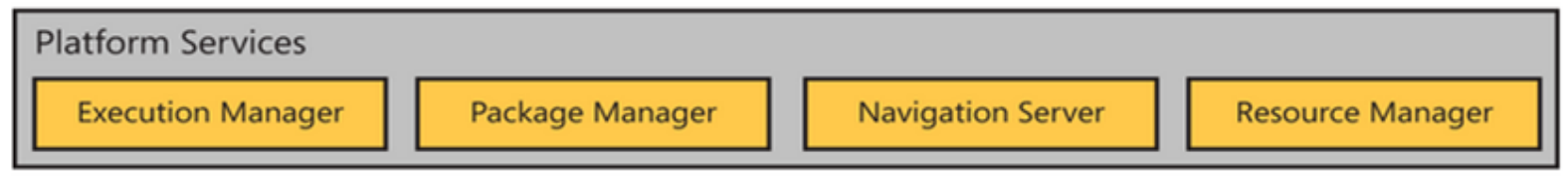

\begin{tabular}{|c|c|c|c|}
\hline \multicolumn{4}{|l|}{ Base OS Services } \\
\hline Networking & Storage & Media & Sensors \\
\hline
\end{tabular}

Fig 1: Windows Phone 8 layers

\section{PROPOSED SCHEME}

The project has been developed under the Windows phone platform using Visual Studio 2012 programming environment, Windows phone SDK, Silverlight SDK and C\#. Figure 1 describes the design of a typical Native App (such Yoruba Bible); a native application made up of Data layer, ViewModel layer, Presentation layer and Application IsolatedStorage layer.
Developing a native Windows phone app, any of the Microsoft .NET languages can be used (such as C\#, VB.net, $\mathrm{F} \#$ and so on), the code written in any of these languages is compiled into an executable binary code and also packaged as ".xap" as show in figure 2. The xap is the format file that is to be published on Windows Phone Market store [3]. The figure 3 illustrates the proposed architecture of the Yoruba Bible app design

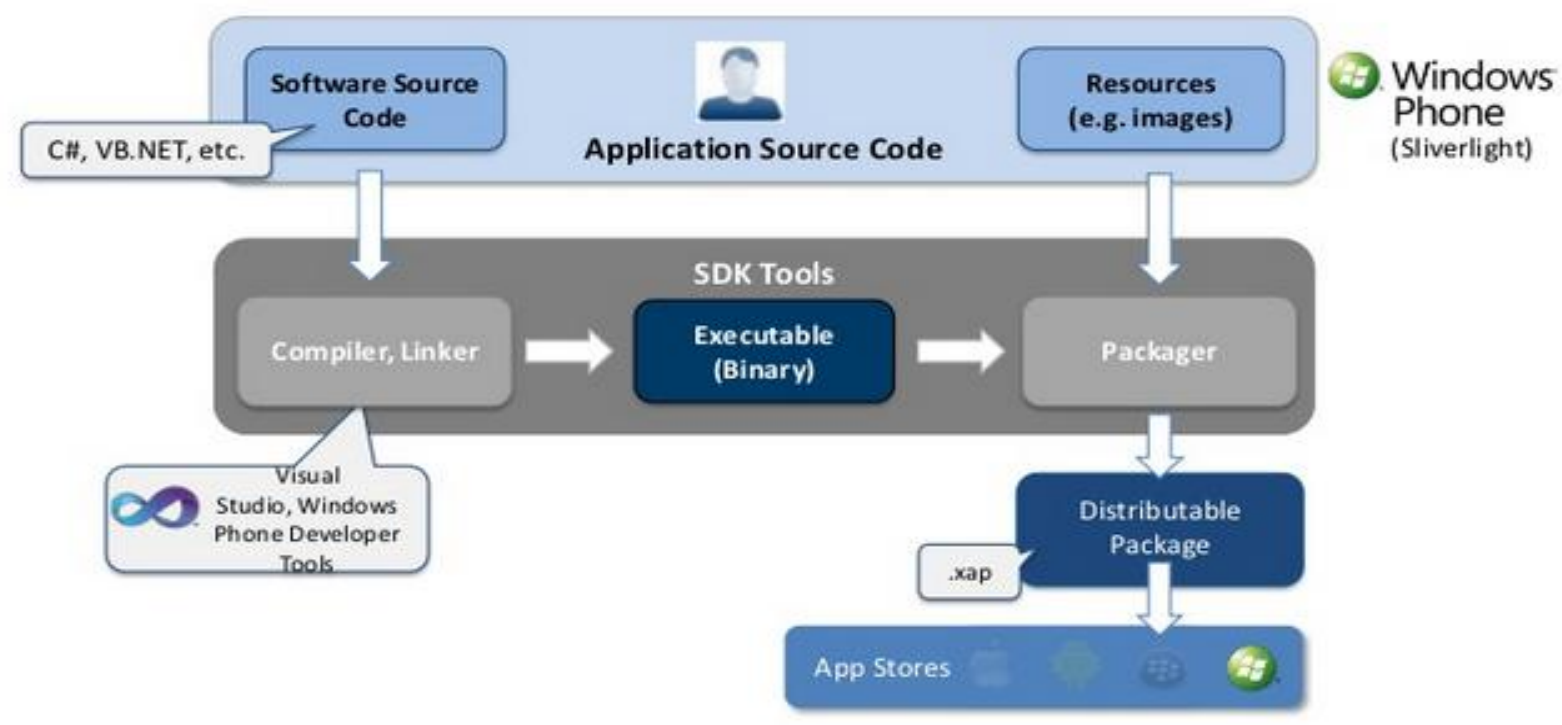

Fig 2: Native Application Development[3] 


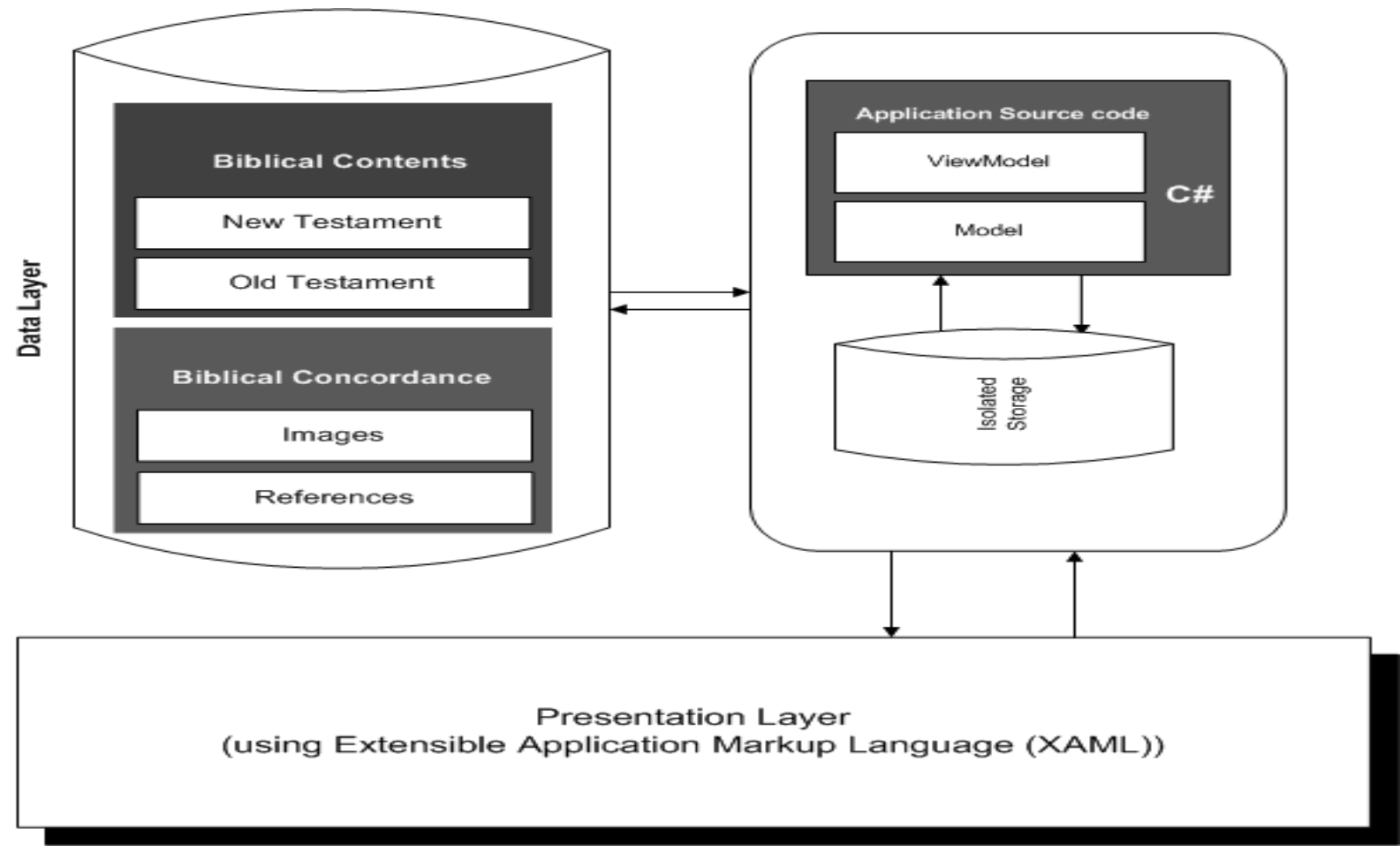

Fig 3: The Architecture of the Yoruba Bible app design

\subsection{Data Layer}

This is a layer in software development which provides simplified access to data storage and it is also responsible for providing data sources. The data layer of the Yoruba Bible app consists of the Bible scriptural content and the bible concordance content. The bible scriptural content contains 66 classes of the bible books, each of these classes return a strings of chapter based on the user request. The bible concordance content contains the concordance topics, concordance biblical references, concordance images

\subsection{View Model Layer}

The business Logics that provides data binding targets for the presentation layer. In many cases, the view model exposes the model directly, or provides members that wrap specific model members. The view model can also define members for keeping track of data that is relevant to the UI but not to the model, such as the display order of a list of items.

\subsection{Presentation Layer}

The presentation layer contains the components that implement and display the user interface and manage user interaction. This layer include controls for user input and display, in addition to components that organize user interaction[4]. The presentation layer acts as an interactive interface between users and the system. It is implemented on the client devices and its responsibility is to deal with the dialog between users and the system. The client devices are primarily categorized as smart mobile phones such as Windows Phone, Windows Surface, blackberry, i-phones, Tablet PCs and so on.

\subsection{Application Isolated Storage}

Isolated storage is a data storage mechanism that provides isolation and safety by defining standardized ways of associating code with saved data. Standardization provides other benefits as well. With isolated storage, code no longer needs unique paths to specify safe locations in the file system and data is protected from other applications that only have isolated storage access. [5]

\subsection{Application Design Layout}

The diagram below shows the design layout of the Yoruba Bible App 


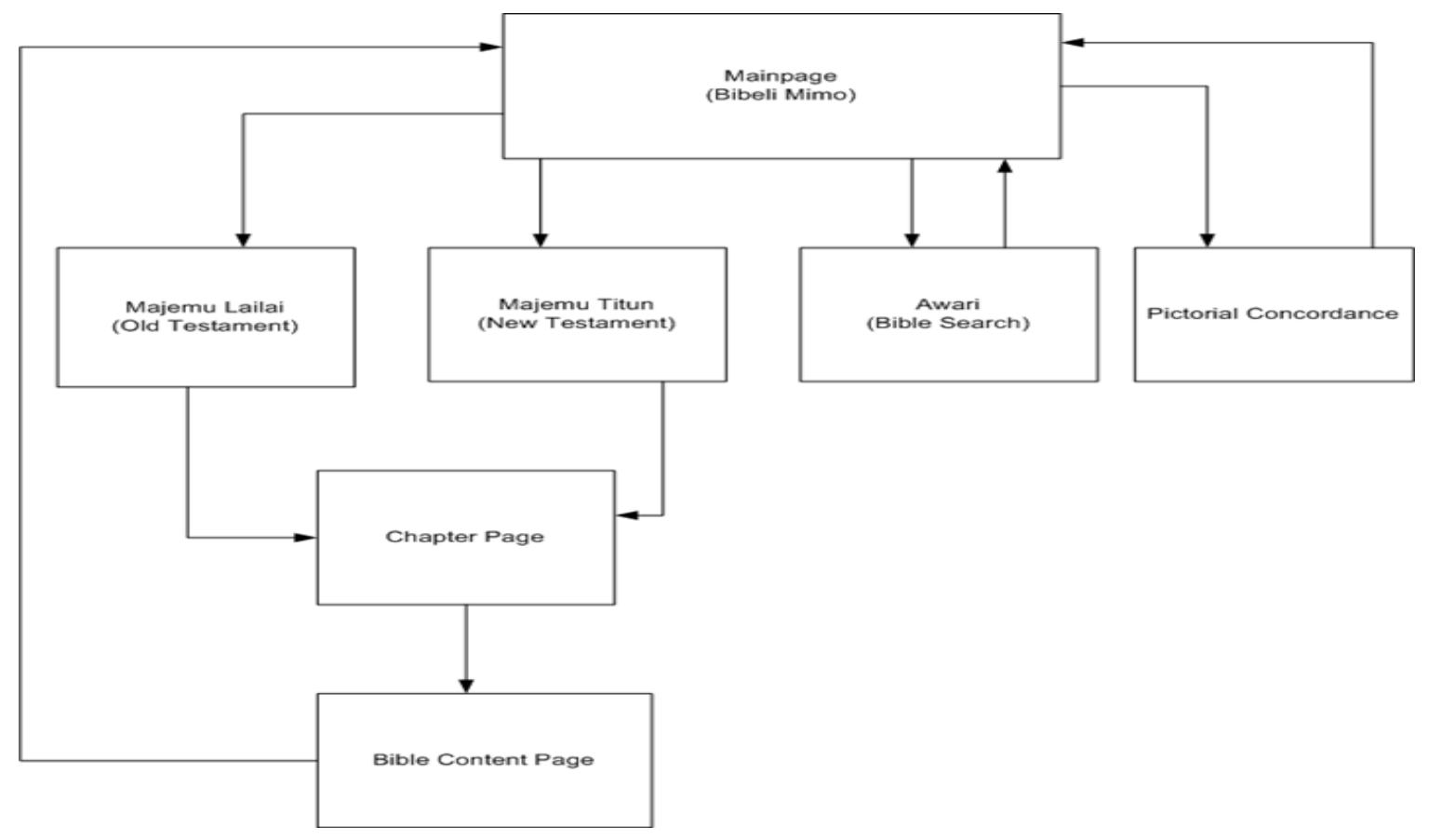

Fig 4: The Design Layout of the Yoruba Bible App

\section{CONTRIBUTION TO KNOWLEDGE AND RECOMMENDATION}

This project work is expected to enhance the understanding of Yoruba language, promote Yoruba people origin and culture and also to provide a good and ease accessibility of the bible in an indigenous language.

The application can annex cloud storage capability (hosting the audio, picture and some minor text files on a cloud storage like Window Azure) and also making the application available on most Smartphone platforms in order for wider range of usage.

\subsection{LIMITATIONS OF THE RESEARCH}

Aside that the interested user must have internet access or data plan subscription to download the app, the following are the limitations of this project work;

- A user must possess a windows phone.

\subsection{APPLICATION SCREEN SHOT}

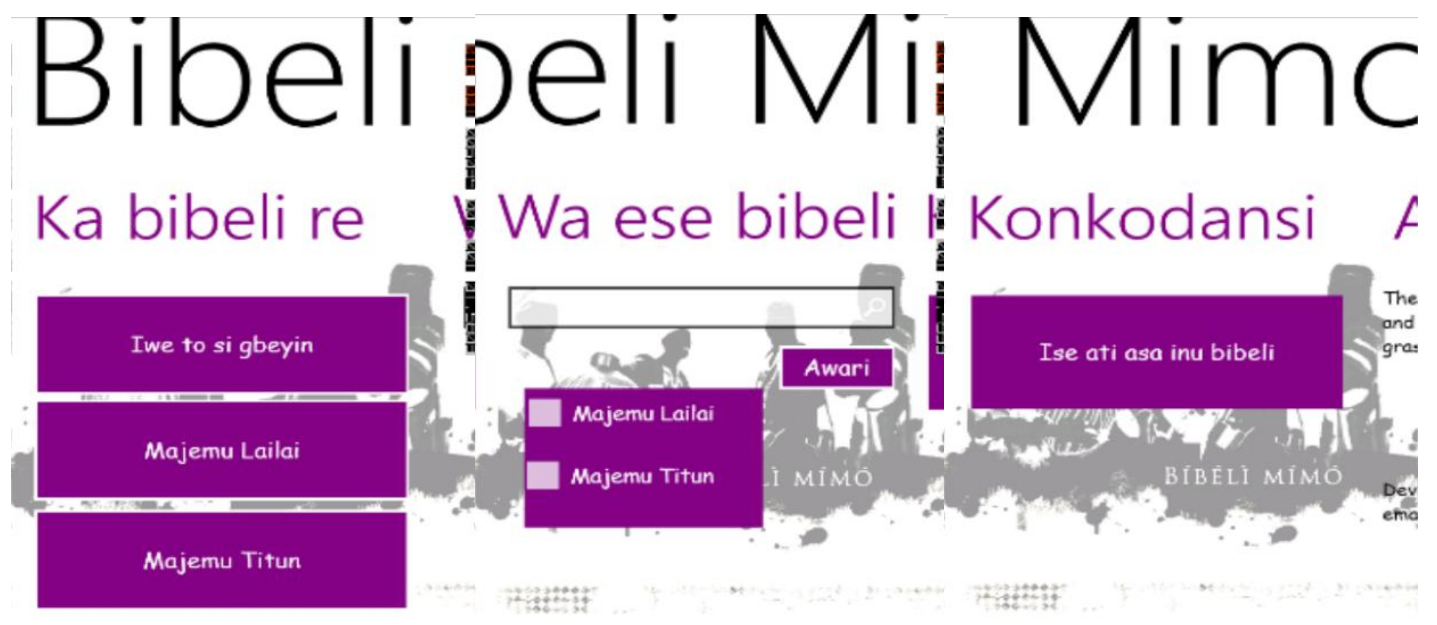

Fig 5: The home page of the yoruba bible app 


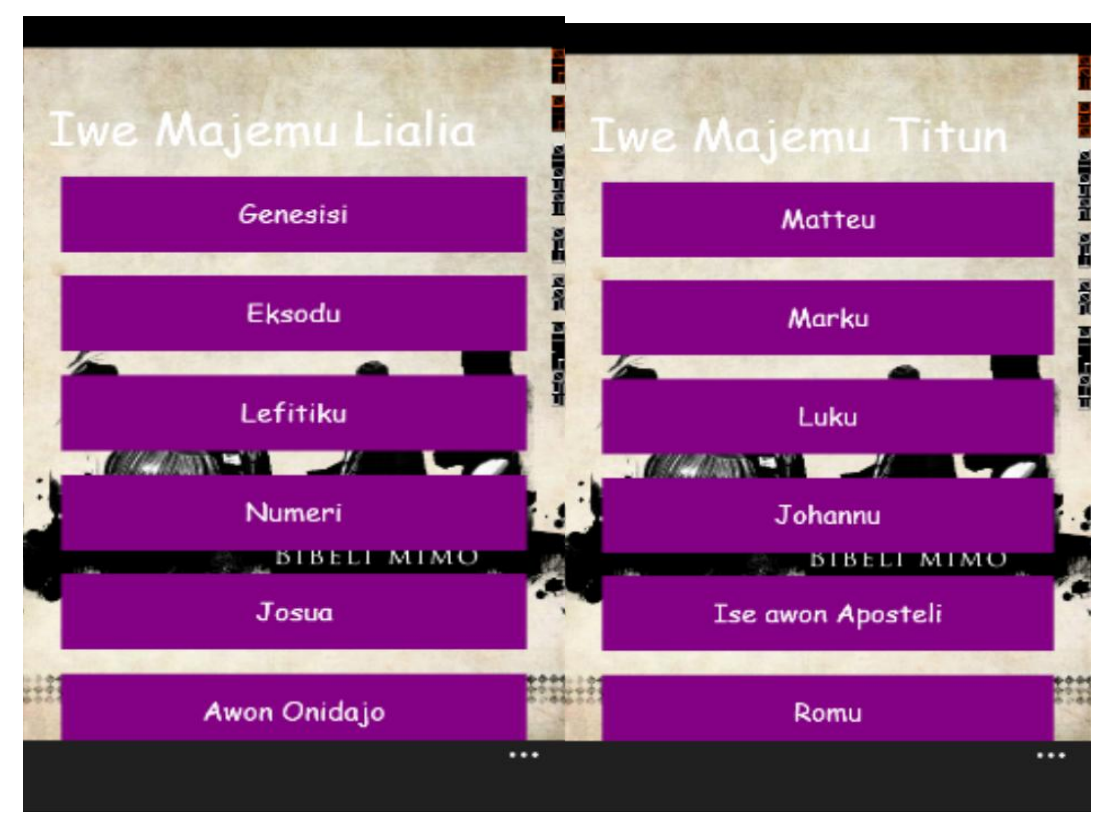

Fig 6: Figure showing the old and the new testament

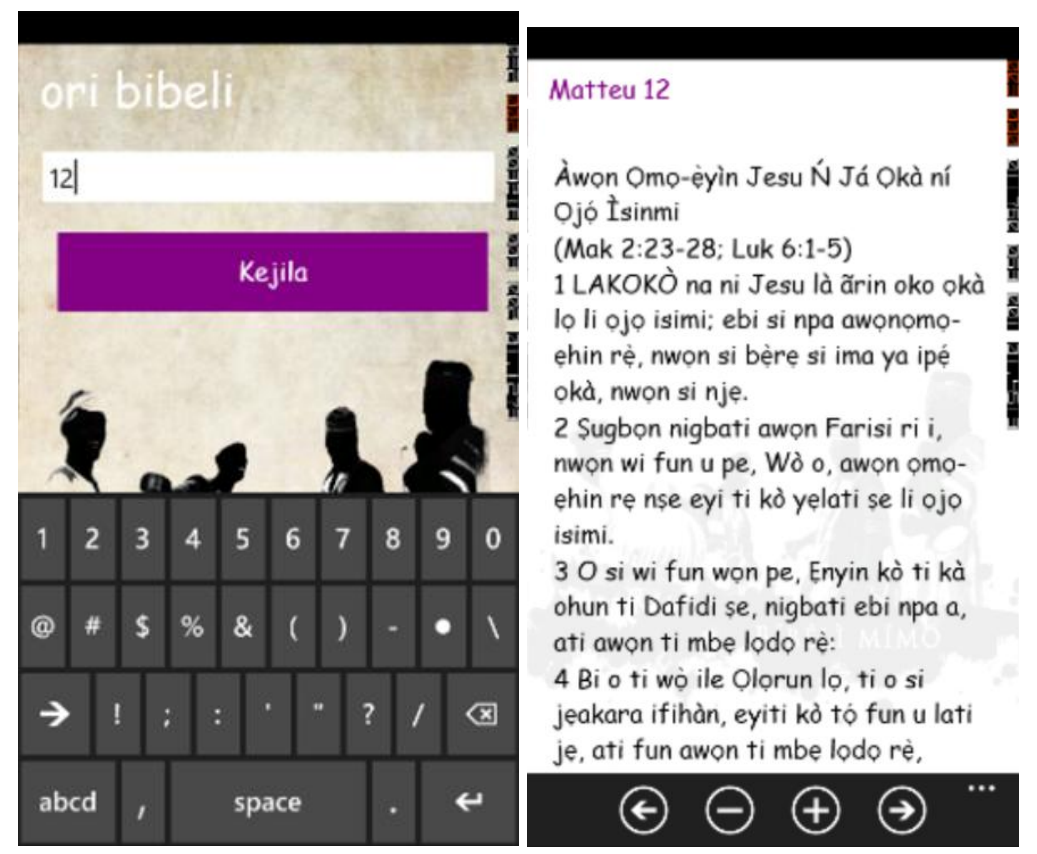

Fig 7: Bible Content Page

( This page contain the list of chapters of the book of the bible clicked by the user, it can also be filtered by typing the figure of the chapter in the textbox above the chapters ) 


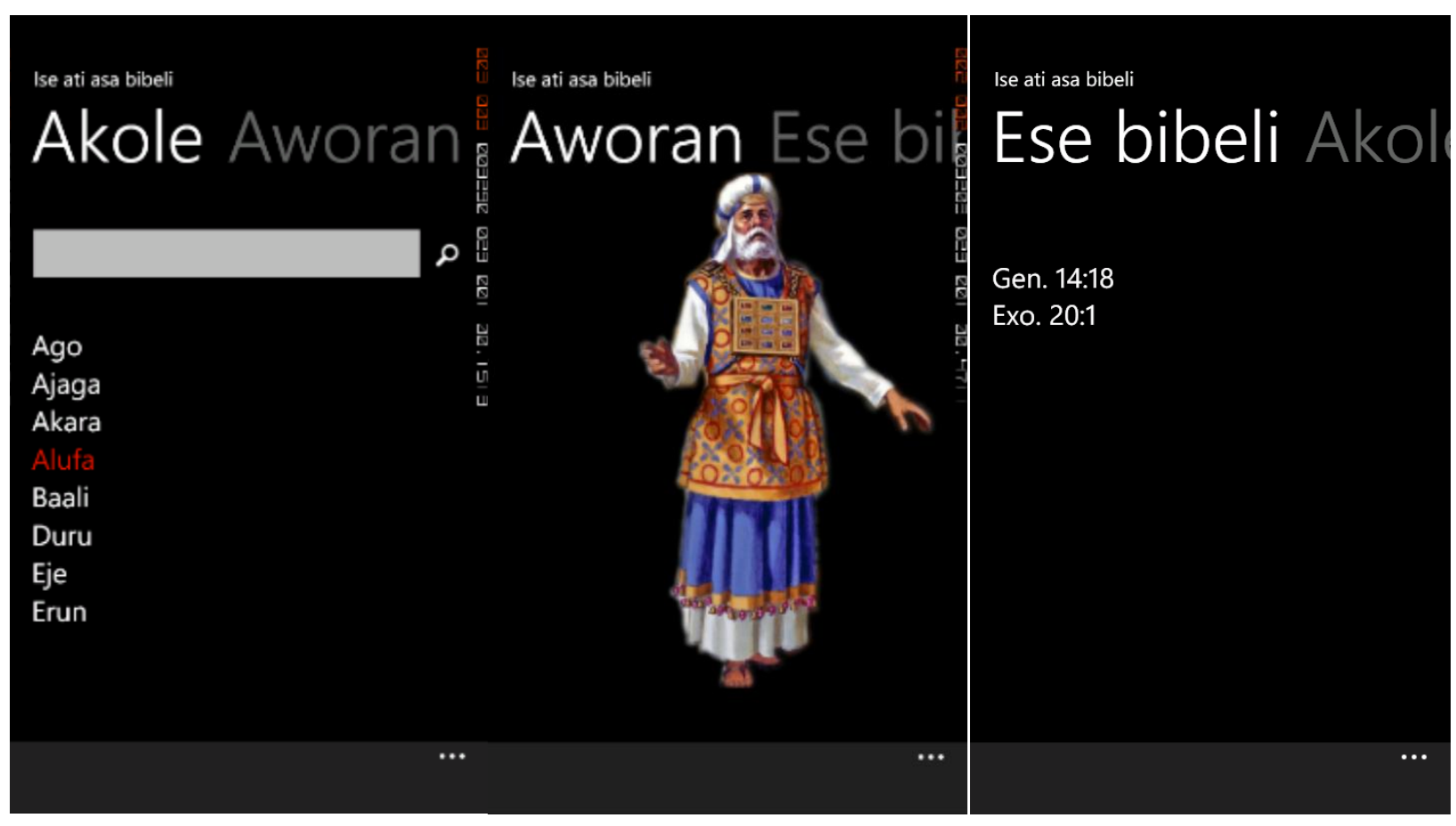

Fig 19: Pictorial concordance and the biblical references.

\section{CONCLUSION}

While there are many versions of bible in the official language across many countries, most African languages don't have any version in their language or dialect. Yoruba bible app provides a bible version in one of the most popular African language, thereby promoting the language and in turn the country and giving access to people who might not comprehend the English version of the bible well enough.

\section{REFERENCES}

[1] Smartphone. (2013, May 30). Retrieved June 13, 2013, from Wikimedia: http://en.wikipedia.org/wiki/Smartphone

[2] O'Reilly Media, I. (2013). Vision and architecture. Retrieved March 4, 2014, from chimera labs oreilly: http://chimera.labs.oreilly.com/books/1234000001853/ch 01.html\#_platform_stack

[3] SlideShare. (2014). native web or hybrid mobile app development. Retrieved March 3, 2014, from SlideShare: http://www.slideshare.net/WorkLightInc/native-web-orhybrid-mobile-app-development-webinar

[4] Microsoft. (2013). Presentation Layer. Retrieved July 10, 2013, from MSDN: http://msdn.microsoft.com/enus/library/ee658081.aspx

[5] Microsoft. (2013). Isolated Storage. Retrieved July 10, 2013, from MSDN: http://msdn.microsoft.com/enus/library/bdts8hk0(v=vs.80).aspx 